Supporting information on es048820p

\title{
Quantifying the Contribution of Different Sorption Mechanisms for 2,4- Dichlorophenoxyacetic Acid Sorption by Several Variable-Charge Soils
}

\author{
Seunghun Hyun and Linda S. Lee
}

Fig. S1. The fractions of hydrophilic sorption $\left(f_{\mathrm{Hphilic}}\right)$ and specifically anion exchange $\left(f_{\mathrm{AE}}\right)$ are shown for several organic acids versus chemical $\mathrm{p} K_{\mathrm{a}}$. The solid line in figures $\mathrm{A}$ and $\mathrm{B}$ is a linear regression fit of the chlorinated phenol data and the dotted line is an extrapolation. The

solid line in $\mathrm{C}, \mathrm{D}, \mathrm{E}$, and $\mathrm{F}$ is a line intersecting the two data points for PCP and prosulfuron and the dotted line is an extrapolation. CP denotes chlorinated phenol with $\mathrm{P}, \mathrm{Te}, \mathrm{T}$, and D denoting penta, tetra, tri, and di-chloro groups, respectively. -page S2 

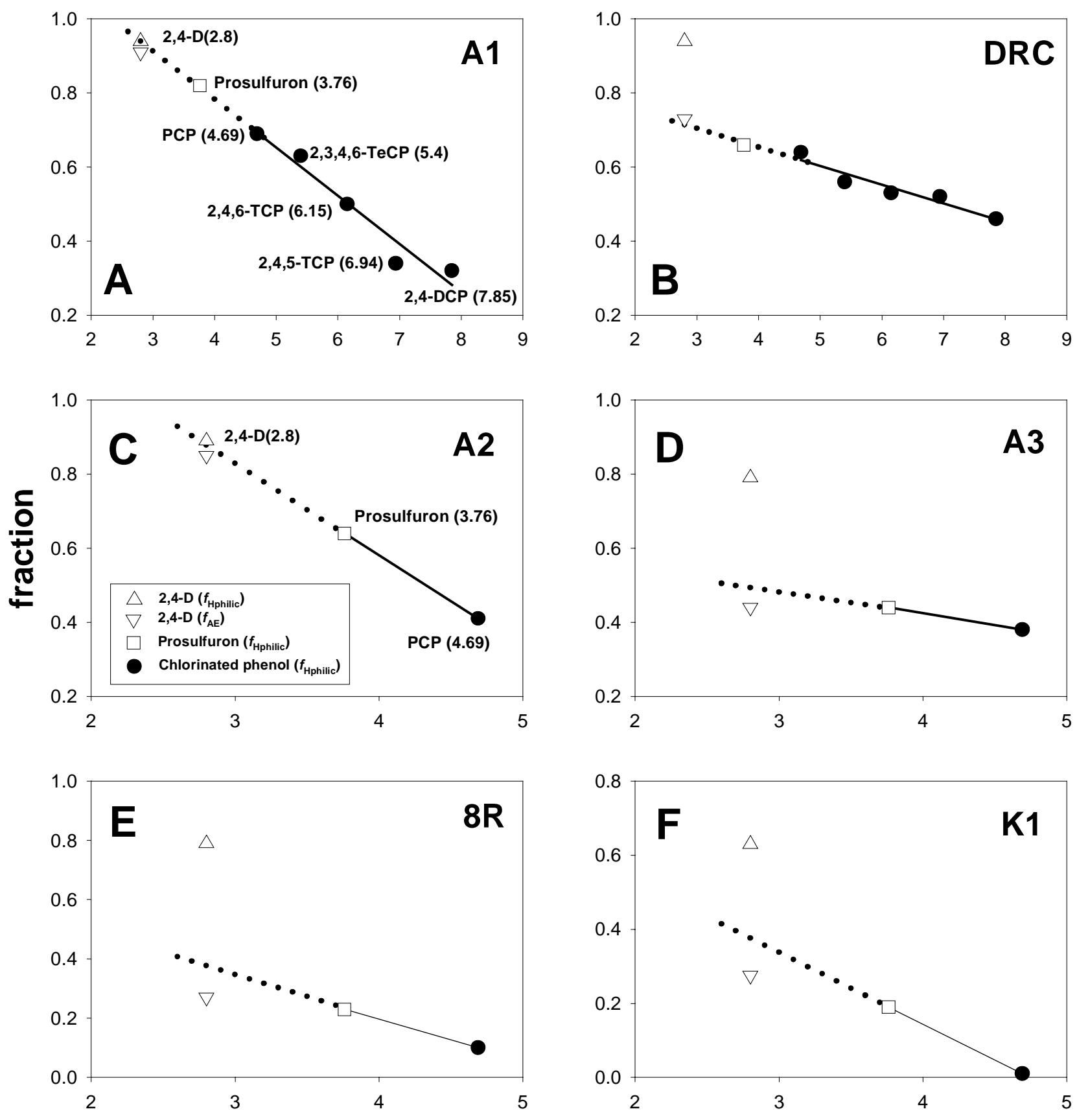

\section{$p K_{a}$ of organic acids}

Fig. S1. The fractions of hydrophilic sorption $\left(f_{\text {Hphilic }}\right)$ and specifically anion exchange $\left(f_{\mathrm{AE}}\right)$ are shown for shown for several organic acids versus chemical $\mathrm{p} K_{\mathrm{a}}$. The solid line in figures $\mathrm{A}$ and $\mathrm{B}$ is a linear regression fit of the chlorinated phenol data and the dotted line is an extrapolation. The solid line in $\mathrm{C}, \mathrm{D}, \mathrm{E}$, and $\mathrm{F}$ is a line intersecting the two data points for $\mathrm{PCP}$ and prosulfuron and the dotted line is an extrapolation. $\mathrm{CP}$ denotes chlorinated phenol with $\mathrm{P}, \mathrm{Te}, \mathrm{T}$, and D denoting penta, tetra, tri, and di-chloro groups, respectively. 